\title{
WHY 'GALACTIC' GAMMA-RAY BURSTS MIGHT DEPEND ON ENVIRONMENT: BLAST WAVES AROUND NEUTRON STARS
}

\author{
Martin J. Rees ${ }^{1}$, Peter Mészáros ${ }^{2}$, and Mitchell C. Begelman ${ }^{3} 4$
}

\section{ABSTRACT}

Although galactic models for gamma-ray bursts are hard to reconcile with the isotropy data, the issue is still sufficiently open that both options should be explored. The most likely 'triggers' for bursts in our Galaxy would be violent disturbances in the magnetospheres of neutron stars. Any event of this kind is likely to expel magnetic flux and plasma at relativistic speed. Such ejecta would be braked by the interstellar medium (ISM), and a gamma-ray flash may result from this interaction. The radiative efficiency, of this mechanism would depend on the density of the circumstellar ISM. Therefore, even if neutron stars were uniformly distributed in space (at least within 1-2 kpc of the Sun), the observed locations of bursts would correlate with regions of above-average ISM density.

\section{INTRODUCTION}

Before there was any firm evidence on the isotropy of classical gamma-ray bursts (GRBs), the most plausible interpretations involved magnetospheres of neutron stars within our Galaxy. Indeed, on the basis of general theoretical plausibility, many people would have bet strongly against a cosmological interpretation. The remarkable isotropy discovered by the BATSE experiment (together with the 'flatter than Newtonian' counts) clearly shifts the odds substantially. If one lays aside theoretical preconceptions, the cosmological interpretation may now seem strongly favoured. However, Bayesians who allow their assessment to be influenced by some prior view of the relative plausibility of the alternative hypotheses may now find the arguments quite evenly balanced. For example, we may think the isotropy is 100 times easier to account for in a cosmological than in a galactic model; however, if we previously would have bet 100 to 1 in favour of a galactic origin, we end up betting 'evens'. This is our rationale for continuing to consider both options.

If they are not cosmological, GRBs would most likely populate a relativcly nearby region of the Galactic disk, at distances $\$(1-2)$ kpc. [As noted by other speakers, 'halo' models entail (at least in a mild form) many of the same

1 Institute of Astronomy, Madingley Road, Cambridge CB3 OHA, England.

2 Pennsylvania State University, 525 Davey Lab, University Park, PA 16803.

3 Joint Institute for Laboratory Astrophysics, University of Colorado and National Institute of Standards and Technology, Boulder, CO 80309

- Department of Astrophysical, Planetary and Atmospheric Sciences, University of Colorado, Boulder, CO 80309.

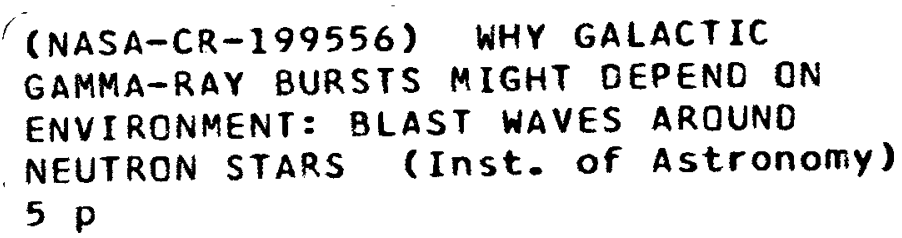


theoretical problems as cosmological models]. The bursts could then be due to violent disturbances in the magnetospheres of neutron stars. ${ }^{1,2}$ Any disturbance of the kind proposed is also likely to expel magnetic flux and plasma into the interstellar medium (ISM) surrounding the neutron star, possibly at relativistic speed. The new point we wish to discuss here is that the blast wave driven into the ISM by a magnetospheric disturbance could also produce a flash of gamma-rays with the characteristics observed to be typical of GRBs. We briefly summarise here the physics of the interaction between relativistic ejecta from a neutron star and the ambient interstellar medium. Fuller details are in reference (3).

\section{RADIATIVE PROPERTIES OF BLAST WAVES}

Suppose an amount of energy $E_{0} \sim 10^{39} E_{30}$ ergs is impulsively released from a neutron star magnetosphere into a medium of number density $n(r) \mathrm{cm}^{-3}$, where $r$ is the distance from the source of the energy. (The time structure and duration of bursts may be partly due to a more complicated pattern of energy release.) The initial energy produces a highly relativistic fluid, with Lorentz factor $\eta$, if the mass $M_{0}$ initially released along with the energy satisfies $E_{0} / M_{0} c^{2} \equiv \eta \gg 1$. After an amount $\eta^{-1} M_{0}$ of external mass has been swept up a blast wave forms ahead of the ejecta, which starts to decelerate. In this decelerating regime, if radiation were inefficient the bulk Lorentz factor of the blast wave, after having reached the value $\Gamma \simeq \eta$, would vary with radius according to

$$
\Gamma \sim\left(\frac{3 E_{0}}{4 \pi m_{p} c^{2} n r^{3}}\right)^{1 / 2} .
$$

The blast wave, however, may radiate away enough of its energy in a sufficiently short time scale to be of interest for explaining GRBs. This can occur, for example, if the magnetic energy density is amplified behind the shock front (due to turbulent shear, etc.) to a significant fraction $(\lambda)$ of equipartition with respect to the shocked ambient gas, or in a reverse shock; ${ }^{4,8}$ Compton losses can also be important.

In the comoving frame, the magnetic field is given by

$$
B^{\prime} \sim 0.3 \lambda^{1 / 2} n^{1 / 2} \Gamma \mathrm{G} .
$$

The highest efficiency is obtained if the gamma rays are synchrotron radiation. To produce synchrotron photons of observed (Doppler-boosted) energy $\varepsilon_{\mathrm{MCV}}$ $\mathrm{MeV}$ requires that electrons be accelerated to random Lorentz factors (in the fluid frame) $\gamma$ such that

$$
\gamma \Gamma \sim 2.6 \times 10^{\top}(\lambda n)^{-1 / 4} \varepsilon_{M e V}^{1 / 2} .
$$

This can be accomplished, in principle, by Fermi accleration at the strong shock front. ${ }^{6}$ For blast wave radii $Z 10^{13} \mathrm{~cm}$ and typical interstellar conditions $n \sim 1$ $\mathrm{cm}^{-3}$, synchrotron radiation at energies above $1 \mathrm{MeV}$ can be highly efficient. ${ }^{3}$ 
If we assume that shock acceleration to a Lorentz factor $\gamma$ requires $100 \zeta_{2}$ gyro-orbital times, then the maximum synchrotron photon energy coming from the blast wave varies according to $\varepsilon_{\operatorname{maz}} \sim 0.4 \zeta_{2}^{-1} \Gamma \mathrm{MeV}$. Note that $\varepsilon_{\operatorname{maz}}$ depends on the highly uncertain shock acceleration rate through $\zeta$. Photons of energy $\varepsilon$ will come predominantly from inside the radius at which $\varepsilon \sim \varepsilon_{\max }$, i.e., where the blast wave has slowed to $\Gamma \sim 2.5 \zeta_{2} \varepsilon_{\mathrm{MeV}}$. If $E \sim 10^{30} E_{30} \mathrm{erg}$, this radius is given by $r_{\max } \sim 3 \times 10^{12}\left(E_{30} / n \zeta_{2}^{2} \varepsilon_{M \mathrm{eV}}^{2}\right)^{1 / 3} \mathrm{~cm}$, corresponding to a maximum burst duration of $\Delta t_{\max } \sim \mathrm{r} / \mathrm{c} \Gamma^{2} \sim 160\left(E_{30} / n\right)^{1 / 3}\left(\zeta_{2} \varepsilon_{M} \mathrm{eV}\right)^{-8 / 3}$ s. This estimate suggests that the maximum burst duration might be anticorrelated with observing frequency. Note, however, that the extreme sensitivity to $\zeta$ makes it difficult to extract useful numerical estimates from this formula.

A necessary condition for the blast wave to radiate efficiently at energy $\varepsilon$ is that $r_{\text {rad }}<r_{\max }$, which is equivalent to the condition

$$
n>0.03 E_{30}^{-4 / 5} \lambda^{-0 / 8} \zeta^{8 / 5} \varepsilon_{M \mathrm{CV}}^{2 / 8} \mathrm{~cm}^{-3}
$$

While the numerical values of the parameters in eq. (4) are very uncertain, the condition suggests a correlation between burst efficiency (and therefore detectability) and the density of the ambient ISM.

\section{INFERENCES FROM BURST STATISTICS}

If bursts repeat on a timescale of order $t_{r}$ years, then the local population of bursters comprises of order $10^{3} t_{r}$ neutron stars. Given a Galactic pulsar birthrate ${ }^{7}$ of $\sim 10^{-11} \mathrm{pc}^{-2} \mathrm{yr}^{-1}$, the mean age of a bursting neutron star is $t_{\text {burat }} \sim 10^{7} R_{k p c}^{3}\left(t_{r} / f\right)$ yr $\equiv 10^{10} t_{10} \mathrm{yr}$, where $R_{k p c}$ is the mean distance to bursts in $\mathrm{kpc}$ and $f$ is the fraction of the time during which the deposition of burst energy in the ISM would lead to a detectable burst. Since the dipole spindown time of a pulsar is $\sim 10^{9} P^{2} B_{11}^{-2} \mathrm{yr}$, the typical spin period of neutron stars responsible for the local bursts would be $\sim 3 B_{11} t_{10}$ s. If $t_{10}<3\left(v_{100} / B_{11}\right)^{1 / 2} n_{\infty}^{-1 / 2}$, these pulsars would still be producing wind bow shocks in the ISM, and would not be accreting interstellar gas.

The contact discontinuity between the shocked pulsar wind and the ISM is then located at $r_{W} \sim 10^{13} B_{11}^{-1} t_{10}^{-1} v_{100}^{-1} n_{\infty}^{-1 / 2} \mathrm{~cm}$. This number is smaller than $r_{\max }$ for $1 \mathrm{MeV}$ photons provided that $B_{11} t_{10} n^{1 / 2}>0.3$, suggesting that detectable bursts from blast waves would come primarily from a relatively old population of pulsars, $t_{b u \text { rot }} \gtrsim 10^{\circ} \mathrm{yr}$, and/or from neutron stars passing through denser regions of the ISM. In either case, we estimate $t_{r} / f \gtrsim 100$. Note that, in the simplest interpretation, $f$ would be the volume filling factor of ISM with high enough density to make the blast wave readily detectable. 


\section{DISCUSSION}

We have extended previous ideas about plausible radiation mechanisms for Galactic GRBs, pointing out that relativistic blast waves driven into the ISM by magnetospheric disturbances around neutron stars can yield bursts of gammarays with roughly the observed range of timescales and fluences. Our extremely simple conjectures about the radiative properties of synchrotron emitting blast waves do not reveal the expected spectral properties of such bursts, but they do suggest a plausible correlation between the radiative efficiency at $\mathrm{MeV}$ energies and the density of the ambient medium.

The question of what might trigger gamma-ray bursts in this picture is unresolved. A neutron starquake model or other impulsive events that violently disturb the magnetosphere seem attractive on energetic grounds. The bursts may be due to neutron stars with unusually strong dipole fields, which have been advocated by Duncan and Thompson (these proceedings) in their interpretation of the soft repeaters. Rotational or gravitational energy would be adequate to power numerous bursts per neutron star. As already explained, the stars would not be accreting from the ISM; however impact of comets or asteroids ${ }^{8}$ are further possibilities.

A relatively robust conclusion is that, for any kind of trigger mechanism which involves violently shaking a neutron star magnetosphere, a strong gammaray burst can be generated by interaction of the expanding energy flow (whatever its form) with the ISM. This does not exclude a gamma-ray burst from the magnetosphere itself, but in the light of the evidence for two classes of classical GRBs, ${ }^{9,10}$ one might perhaps attribute the short bursts to the latter mechanism and the long $(t \gtrsim 2 s)$ to the blast wave. The efficiency of this mechanism depends on the ISM (which may introduce longer timescale variability), so the blast wave component would be specially dominant for bursts occurring in regions where the ISM has reasonably large density (e.g. clouds, not necessarily molecular).

[We are discussing primarily the "classical" bursts rather than the soft repeaters. However it is worth noting parenthetically that the one feature of the latter which causes problems for Duncan and Thompson's model (these proceedings) is the sharp initial spike on the famous "March 5th" event. If the event released some relativistic plasma, this intense and short-lived percursor burst would be accounted for if $\Gamma \gtrsim 500$. If the ejection were directional, its energy could of course be well below the value of $10^{44}$ ergs inferred on the basis of isotropic emission.]

We recall that old pulsars would be expected to have a smooth distribution in the galaxy, constituting a halo population or a disc population with a large scale height. If the bursts came from $\gtrsim 10 \mathrm{kpc}$ distances, one would expect a strong systematic concentration towards the Galactic Centre; on the other hand, if the burst distances are only $1 \mathrm{kpc}$ or less and their distribution directly traced that of the old neutron stars, the non-uniformities revealed by $V / V_{m}$ would be 
perplexing. A $\log N-\log S$ slope flatter than $3 / 2$ at low fluences can be easily understood in terms of a dropoff in the number of sources beyond a local high density excess associated with our immediate neighbourhood. However, it would seem a bit of a coincidence that the anisotropy should be so small relative to the deficit from Euclidean counts - this would imply that we were relatively near the centre of a kpc-scale region where the mean ISM density was higher than outside.

This model, based on a local burst population made conspicuous by a denser gaseous environment, would predict that the spatial distribution would be modulated by the highly irregular and structured distribution of the ISM. We could even account for the spiral-arm effects discussed by Quashnock \& Lamb. ${ }^{11}$ If this effect indeed exists, our explanation seems more plausible than attributing all bursts to neutron stars just a few million years old which still remember the spiral arm they came from, since the latter would require a much higher repetition rate.

We acknowledge partial support from NSF grant AST91-20599 and NASA grant NAG5-2026 (MCB), from NASA grant NAGW-1522 (PM) and from the Royal Society (MJR).

\section{REFERENCES}

1. Blaes, O., Blandford, R.D., Goldreich, P., \& Madau, P. 1989, ApJ, 343, 839

2. Ramaty, R., Bonazzola, S., Cline, T.L., Kazanas, D. \& Mészáros, P., 1991, Nature, 287, 122

3. Begelman, M.C., Mészáros, P., \& Rees, M.J. 1993, MNRAS, 265, L16

4. Mészáros, P., Laguna, P , \& Rees, M.J. 1993, ApJ, 415, 181

5. Mészáros, P., Rees, M.J. \& Papathanassiou, H., 1994, ApJ, submitted

6. Ellison, D.C. and Reynolds, S.P., 1991, ApJ, 382, 242

7. Narayan, R. and Ostriker, J.P., 1990, ApJ, 352, 222

8. Harwit, M. and Salpeter, E.E., 1973, ApJ, 186, L37.

9. Kouveliotou, C., et al, 1993, ApJ, 413, L101

10. Lamb, D.Q., Graziani, C. \& Smith, I., 1993, ApJ, 413, L11

11. Quashnock, J.M. and Lamb, D.Q. 1993, MNRAS (in press). 